ARTICLE OPEN

\title{
2D organic molecular metallic soft material derived from BEDO-TTF with electrochromic and rectifying properties
}

Daniel Suarez ${ }^{1}$, Eden Steven ${ }^{1,2}$, Elena Laukhina ${ }^{30,4}{ }^{3,}$ Andres Gomez $\mathbb{D i D}^{4}$, Anna Crespi ${ }^{4}$, Narcis Mestres ${ }^{4}$, Concepció Rovira ${ }^{3,4}$, Eun Sang Choi ${ }^{1}$ and Jaume Veciana ${ }^{3,4}$

In this article we demonstrate that a migration of iodine species and chemical transformation in a moist environment induced by a voltage-biased Pt electrode is able to alter the color and degree of charge transfer in a layer of the 2D organic molecular metal $(\text { BEDO-TTF })_{2.4} l_{3}$ [BEDO = bis(ethylenedioxy)tetrathiafulvalene] self-assembled at the surface of a polycarbonate film. These effects produce a reversible electrochromic behavior of the layer with low operating voltages and fast operation times. Adjuvant with electrochromism, this flexible material exhibits rectifying behavior whose I-V curves are dependent on the voltage sweep directions. These results open new possibilities for the design and fabrication of organic flexible materials for soft electrochromic and rectifying components. The easy working principle ensures reliability, low power consumption, and versatility through its implementation into simple devices. Such working principle has been confirmed by temperature dependent resistance measurements, X-Ray, EDX-SEM, and conducting-AFM studies.

npj Flexible Electronics (2018)2:29; doi:10.1038/s41528-018-0041-1

\section{INTRODUCTION}

Wearable electronics, cell phones, smart windows, sensors, and wireless communication devices ${ }^{1-4}$ need mechanically flexible electronic components with electrochromic ${ }^{1-3}$ and rectifying diode properties. ${ }^{5}$ In this context, the development of lightweight flexible organic conducting materials with electrochromic and rectifying properties has a great technological interest for their applications as electronic papers, smart windows, mirrors or glasses as well as flexible displays for cell phones. ${ }^{1,6-9}$

Different strategies have been proposed to fabricating such flexible electronic components which all are based on thin-film technologies. One of these strategies uses thin-films of electrochromic conducting polymers, such as poly(3,4-ethylenedioxythiophene) (PEDOT) and its derivatives. ${ }^{10,11}$ It is shown that these polymers are very promising candidates as flexible electrochromic materials due to their high coloration efficiency, low band-gap, mechanical and electrochemical stability, and their availability in diverse colors. ${ }^{10}$ However, further investigations to enhance the optical contrast and the long-term stability of conducting polymer-based electrochromic devices are still needed. Some of the recently reported flexible Schottky diodes are made of crystalline silicon microparticles, which require complex and expensive processing steps. ${ }^{12}$ Another strategy is based on thinfilms of semiconducting inorganic materials, like indium-galliumzinc-oxide, which is deposited on flexible plastic substrates. ${ }^{5}$ This type of hybrid material has excellent performances. However, it should be noted that organic polymers and inorganic materials have very different mechanical elastic constants. This mismatch may lead to durability issues with this type of hybrid materials. ${ }^{13}$ The similarity of the elastic properties of layered components is desired for fabricating robust flexible electronic devices. Therefore, there is still a lot of room for improvement in regard to the longterm stability of the electroactive layers and their adhesion to plastic flexible substrates.

In previous years some groups have developed a series of allorganic layered thin-films with tunable conducting properties that range from semiconducting, metallic, to superconducting. ${ }^{14-16}$ These layered thin-films are composed of an active layer of crosslinked organic conductor microcrystals that are directly grown on top of an insulating polymeric thin-film. These bi-layered (BL) materials are fabricated with a simple and scalable procedure, enabling the production of flexible, transparent, conformable, moldable, transferable, stretchable, and robust electronic materials with active layers of organic conductors that can be chosen " $a$ la carte" and that are stable for several years at ambient conditions. Remarkably, these novel materials exhibit physical properties $^{17-22}$ difficult to replicate with silicon or any other inorganic materials as well as with conducting polymers. ${ }^{23}$ Thus, they are appealing to be one of the low-cost alternatives to today's silicon-based devices. ${ }^{24}$ Indeed, these flexible, durable, and lightweight BL materials have been used to fabricate electrical circuits and various sensors for detecting pressures, strains or temperature changes. ${ }^{17,18,25,26}$

The above mentioned facts stimulated us to develop organic flexible material with electrochromic and rectifying properties based on such conducting BL films. In particular we focus our interest on BL films with an active layer of metallic (001) oriented microcrystals of (BEDO-TTF) $2_{2.4} l_{3} \quad$ [BEDO-TTF $=$ bis(ethylenedioxy) tetrathiafulvalene $]^{27-30}$ that is directly grown on top of a thin polycarbonate substrate (Fig. 1a). The selection of this particular type of BL film for such purposes is ideal due to three reasons.

\footnotetext{
${ }^{1}$ Department of Physics, National High Magnetic Field Laboratory Florida State University, 1800 East Paul Dirac Drive, Tallahassee, FL 32310, USA; ${ }^{2}$ Emmerich Education Center, Jakarta 14450, Indonesia; ${ }^{3} \mathrm{CIBER}$ de Bioingeniería, Biomateriales y Nanomedicina (CIBER-BBN), Madrid, Spain and ${ }^{4}$ Institut de Ciencia de Materials de Barcelona (ICMAB-CSIC), Campus UAB, Bellaterra 08193, Spain

Correspondence: Eden Steven (esteven@emmerich.co.id) or Elena Laukhina (laukhina@icmab.es) or Andres Gomez (agomez@icmab.es)
}

Received: 19 January 2018 Revised: 28 August 2018 Accepted: 4 September 2018

Published online: 08 November 2018 


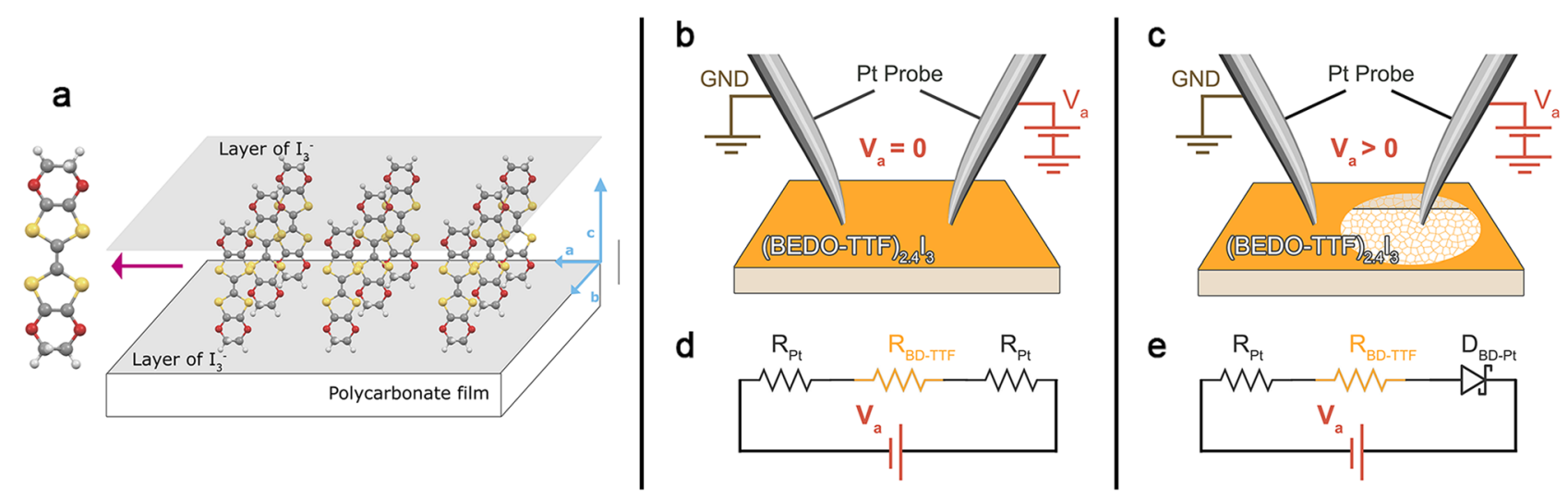

Fig. 1 (BEDO-TTF $)_{2.4} \mathrm{l}_{3}$ bilayer film and measurement setup model. a Schematic representation of the BL film with metallic layer of (001)oriented (BEDO-TTF) $)_{2.4} \mathrm{l}_{3}$ crystallites: atoms of oxygen and sulfur are indicated as red and yellow balls, respectively. $\mathbf{b}$, $\mathbf{c}$ Scheme of the formation of a diode on the film in a two-point Pt-contact configuration. d, e Electrical model of the top-top contact configuration: $\mathrm{R}_{\mathrm{Pt}}$ is a series resistance model of a metallic contact between tip and sample, $R_{B D-T T F}$ is the resistance of the (BEDO-TTF) $)_{2.4} l_{3}$ film, and $D_{B D-P t}$ denotes the diode-like contact between the tip and the sample

First, unlike most polycrystalline thin films where their properties suffer due to grain boundaries and defects, the metallic triiodide salt of BEDO-TTF system is exceptionally robust. This allows the inheritance of the single-crystal electronic properties even though the active layer is a network of oriented microcrystals at the surface of a thin polycarbonate film. ${ }^{29-31}$ Secondly, the metallic polycrystalline layer of (BEDO-TTF) $2_{2.4} l_{3}$ are formed under ambient conditions (Fig. 1a) via a very simple procedure. ${ }^{22,30,31}$ Finally, a previous report has shown some evidence of colorchanging effects in this system. ${ }^{31}$ It was suggested by Adam $\mathrm{Tracz}^{31}$ that the polycrystalline layer of this metallic and intensively brown-colored salt may be transformed into a layer of colorless (BEDO-TTF) $)_{x}\left[\mathrm{l}\left(\mathrm{H}_{2} \mathrm{O}\right)_{n}\right]$ salt. This transformation occurs when the layer of (BEDO-TTF) ${ }_{2.4} \mathrm{I}_{3}$ of BL films losses the $\mathrm{I}_{3}{ }^{-}$anions when it is in contact with a metal electrode in the presence of water. ${ }^{31}$ It was suggested that the colorless conducting layer is composed of a monoiodide salt, such as (BEDO-TTF) ${ }_{x}\left[I\left(\mathrm{H}_{2} \mathrm{O}\right)_{n}\right]$, in which $\mathrm{I}^{-}$anion is stabilized with hydrogen bonds of $\mathrm{H}_{2} \mathrm{O}^{31,32}$ Consequently, the electrical properties of the monoiodide salt are strongly related to the amount of water and $\mathrm{I}^{-}$incorporated into the anionic layer of the salt. ${ }^{31}$

Due to the above mentioned reasons, we have chosen the polycarbonate/(BEDO-TTF) $)_{2.4} l_{3}$ film as the main candidate for developing a flexible electrochromic and rectifying components. In this article, we demonstrate a verification of the removability of $\mathrm{I}_{3}{ }^{-}$anions in the presence of water as the main mechanism for the discoloration effects. More excitingly, we discovered that the discoloration can be reversed by applying a reverse bias (ESI, Supporting Movie S1 and S2), providing further insights on the $\mathrm{I}_{3}{ }^{-}$ anions interactions with the organic species. Furthermore, rectifying behaviors are also promoted as a result of the modification of the degree of charge transfer. The fast highcontrast electrochromism and rectifying properties of the polycarbonate/(BEDO-TTF) ${ }_{2.4} l_{3}$ provide yet another example of the versatility of organic bilayer films.

\section{RESULTS AND DISCUSSION}

BL films with polycarbonate/metallic layer of (001)-oriented (BEDO-TTF) $)_{2.4} \mathrm{l}_{3}$ crystals (Fig. 1) were prepared and studied under external electrical fields with a wide range of voltage bias and under different humidity conditions. The formation of the metallic (001) oriented layers of (BEDO-TTF) $)_{2.4} l_{3}$ on the BL film was confirmed by X-ray diffraction, low-frequency Raman spectroscopy, conducting AFM, and DC transport measurements.
The X-ray diffraction measurement was first carried out on the conducting layer of the polycarbonate/BEDO-TTF ${ }_{2.4} l_{3}$ bilayer film. The X-ray pattern shows only the peaks that correspond to the reflections of (001) planes of (BEDO-TTF) $)_{2.4} \mathrm{I}_{3}$ crystals (ESI, Figure ESI1). This confirms the relative orientation of the BEDO-TTF molecules as schematically shown in Fig. 1a and Figure ESI1 (inset). At this orientation the molecular conducting layer of BEDOTTF-based radical cations is parallel to the film plane. Likewise, the anionic layer that comprises of the linear triiodide counterions (located at the corners of the unit cell) ${ }^{27}$ is also parallel to the film plane (Fig. 1a). Additionally, the X-ray pattern shows a broad peak at $2 \theta=17.275$ which corresponds to a $d$ value of $5.1331 \AA$ (Figure ESI1). This broad peak may originate from the PC substrate ${ }^{33}$ or from the formation of some amount of the (BEDO-TTF)-based product in a low crystalline state. Next, the low-frequency Raman spectra (ESI, Figure ESI2) exhibits a band at $107 \mathrm{~cm}^{-1}$ that corresponds to the symmetric stretching mode of $\mathrm{I}_{3}{ }^{-}$anions in (BEDO-TTF $)_{2.4} l_{3}$ crystals, ratifying the nature of the counter ion of the salt. ${ }^{30}$ The topographic and current AFM images (ESI, Figure ESI3) reveals that the (BEDO-TTF) $)_{2.4} l_{3}$ layer is composed of elongated plate-like crystallites that form a highly conductive network with ohmic-like behavior. Finally, the four-point temperature dependent resistance of the (BEDO-TTF) $2.4 l_{3}$ film (ESI, Figure ESI4a) also reveals a metallic behavior. We therefore confirmed that the metallic (001)-oriented layer of (BEDO-TTF) ${ }_{2.4} l_{3}$ salt is present at the surface of the polycarbonate films.

To begin our tests, first Pt wires were contacted to the active (BEDO-TTF $)\left._{2.4}\right|_{3}$ layer of the BL films in a two-point top-top configuration (Fig. 1b for the initial configuration and Fig. 1c for transparent state configuration). An electrical model of the top-top structure is provided in Fig. $1 \mathrm{~d}$, for the initial configuration, and Fig. 1e for the transparent state configuration. The setup can be modeled as three series resistors, representing the two metallic interfaces at the Pt-tip and the resistance of the (BEDOTTF) $)_{2.4} l_{3}$ layer. $^{34,35}$ As will be discussed later, when the film is in its pristine state, metallic contacts are established between the Pt wires and the conducting layer. During and after the color transformation, its metallic state is not preserved and a Schottky Barrier Height $(\mathrm{SBH})$ is formed at the contact interface which governs the global transport properties, inducing a rectifying behavior (Fig. 1c, e). ${ }^{36}$

To demonstrate the color transformation of the BL film, a voltage bias was applied under different humidity levels. The transformation was realized when we applied an electric field with a positive voltage bias at relative humidity $(\mathrm{RH})$ levels higher than 

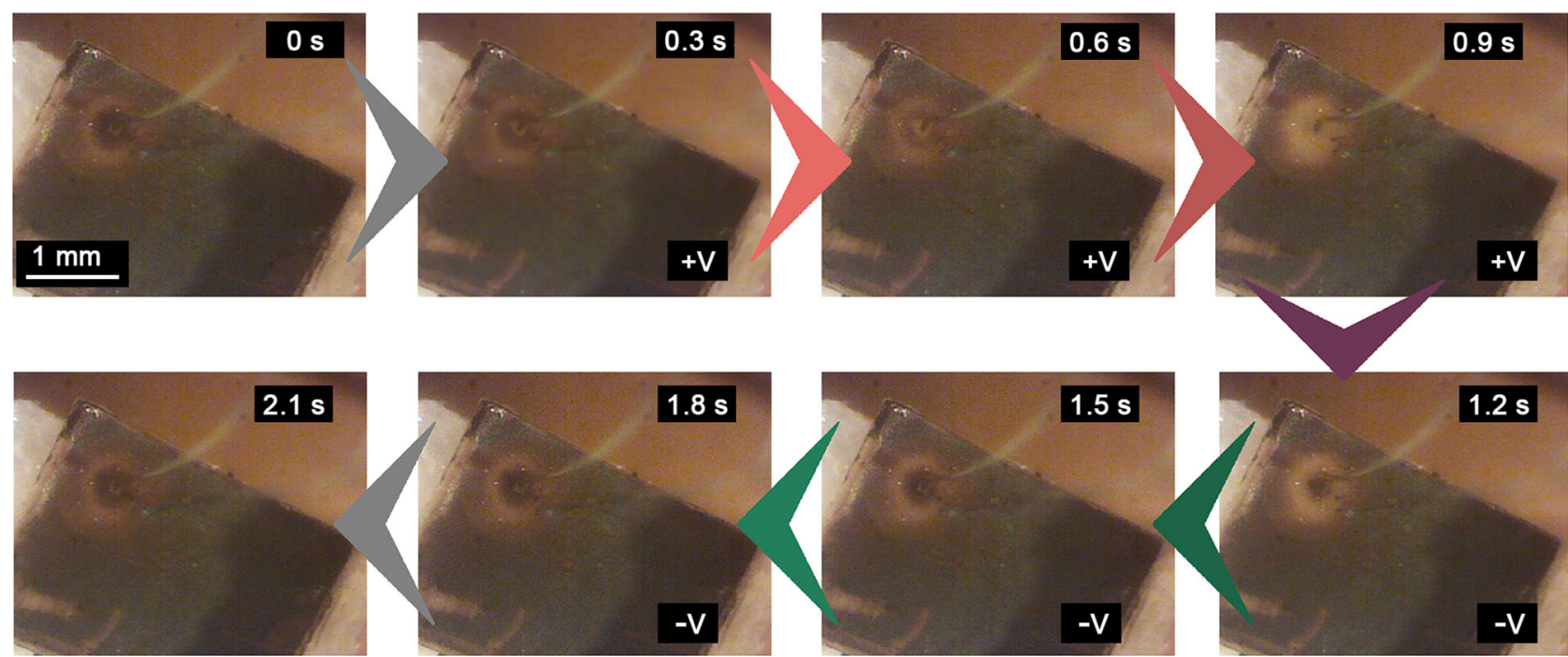

Fig. 2 Snapshots extracted from the Supporting Movie S2 (see ESI). This series of photos demonstrates the time-scale and reversibility of the voltage-dependent discoloration/coloration of the thin conducting layer of the BL film. Applied bias was $+5 \mathrm{~V}$ during the discoloration, and $-5 \mathrm{~V}$ during the recovery
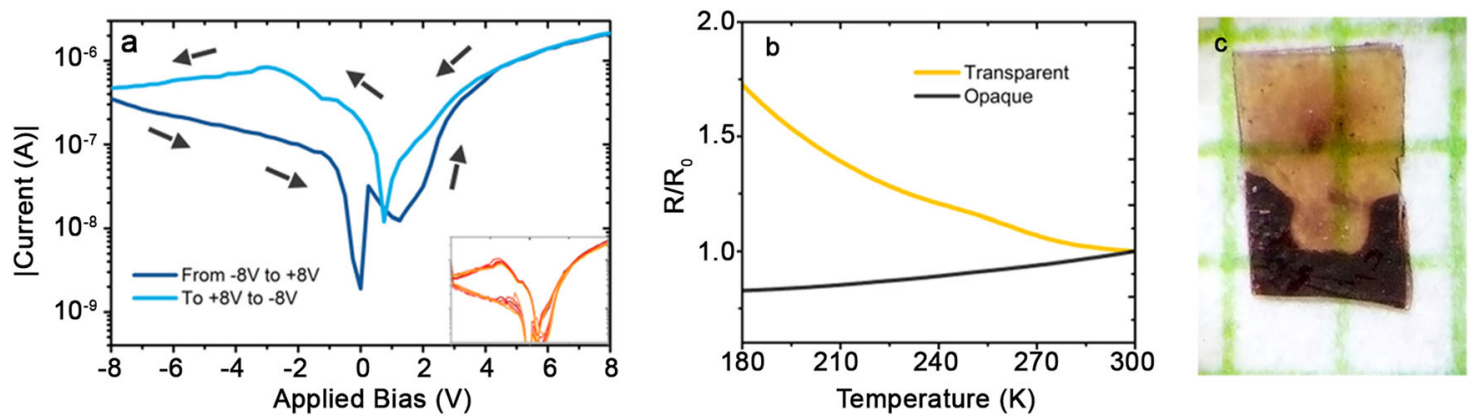

Fig. 3 Correlation between electrochromism and rectifying behavior of BL films. a Cyclic I-V curves showing a diode-like behavior during the discoloration-coloration processes. Inset: eight cyclic I-V curves demonstrating the stability of the system. b Normalized temperature dependent resistance of the transparent (semiconducting) and opaque (metallic) film. c Optical image of the partially discolored film at ambient conditions showing its transparency

$85 \%$ (Fig. 2). A reversible voltage-dependent discoloration process was observed around one of the probe contacts (ESI, Supporting Movie S1 and S2 up to $\pm 8 \mathrm{~V}$ operating voltages). This process is fast in comparison with previous results in other materials. ${ }^{37-39} \mathrm{We}$ also confirmed that the discoloration process did not happened at lower RH levels; so it is clear that the discoloration of the conducting layer of the BL film requires water molecules. To overcome the need of operating at high humidity ambient conditions, we have performed measurements with a simple application of a thin hydrophilic PVA (polyvinyl alcohol) layer on the top of the BL film's surface to increase and keep the water content. This method works remarkably well, allowing the electrochromism to be realized at ambient conditions.

Besides electrochromism, the electrical response of the BL film to cyclic voltages from -8 to $+8 \mathrm{~V}$ (see ESI, Supporting Video S2 at $\pm 8 \mathrm{~V}$ operating voltage) demonstrates a rectifying behavior which is correlated with the voltage-dependent discoloration. As shown in Fig. 3a, after several conditioning cyclic voltage applications ( 10 cycles) (ESI, Figure ESI5), stable I/V curves are achieved. Indeed, the inset of Fig. 3a contains eight overlapped voltage cyclic curves, demonstrating the stability of the rectifying behavior. We note that a huge hysteresis was observed and could be attributed to the contribution of instantaneous current during the chemical processes in the discoloration processes. Despite the hysteresis, it was evident that the rectifying behavior persisted. Preliminary results on other BEDO-TTF bilayer system showed the suppression of such hysteresis and consequently improved the rectifying behaviors. From another perspective, perhaps such a hysteretic effect could potentially be utilized for memory applications. ${ }^{35}$

The origin of the rectifying behavior can be attributed to the classical picture of semiconductor/metal interfaces. To confirm this, we performed temperature dependent resistance measurements of the discolored film after bias applications and compared it to that of the pristine opaque film. Indeed, from the temperature dependent resistance (Fig. 3b), the discolored film is a semiconductor whereas the pristine opaque film is metallic. This difference effectively increases the potential barrier across the electrodes, resulting in a rectifying behavior. Lastly, the room temperature sheet resistance (ESI, Table ESI1) of the discolored film is two orders of magnitude higher than that of the pristine film, indicating a possible redistribution of iodine content of the film.

According to the previous report, ${ }^{31}$ the electrochromic and rectifying properties of the $\mathrm{BL}$ film should originate from the substitution of $I_{3}$ anion with hydrated $I$ anion. To prove this mechanism, the concentrations of sulfur and iodine atoms around the $\mathrm{Pt}$ electrode were examined after the application of positive electric potential (bias). EDX analysis was carried out under SEM to map out the elemental distribution on the BL film (Fig. 4). The following results are obtained: (i) the sulfur content is uniform over the whole studied area (Fig. 4b, d); (ii) the iodine is abundant 

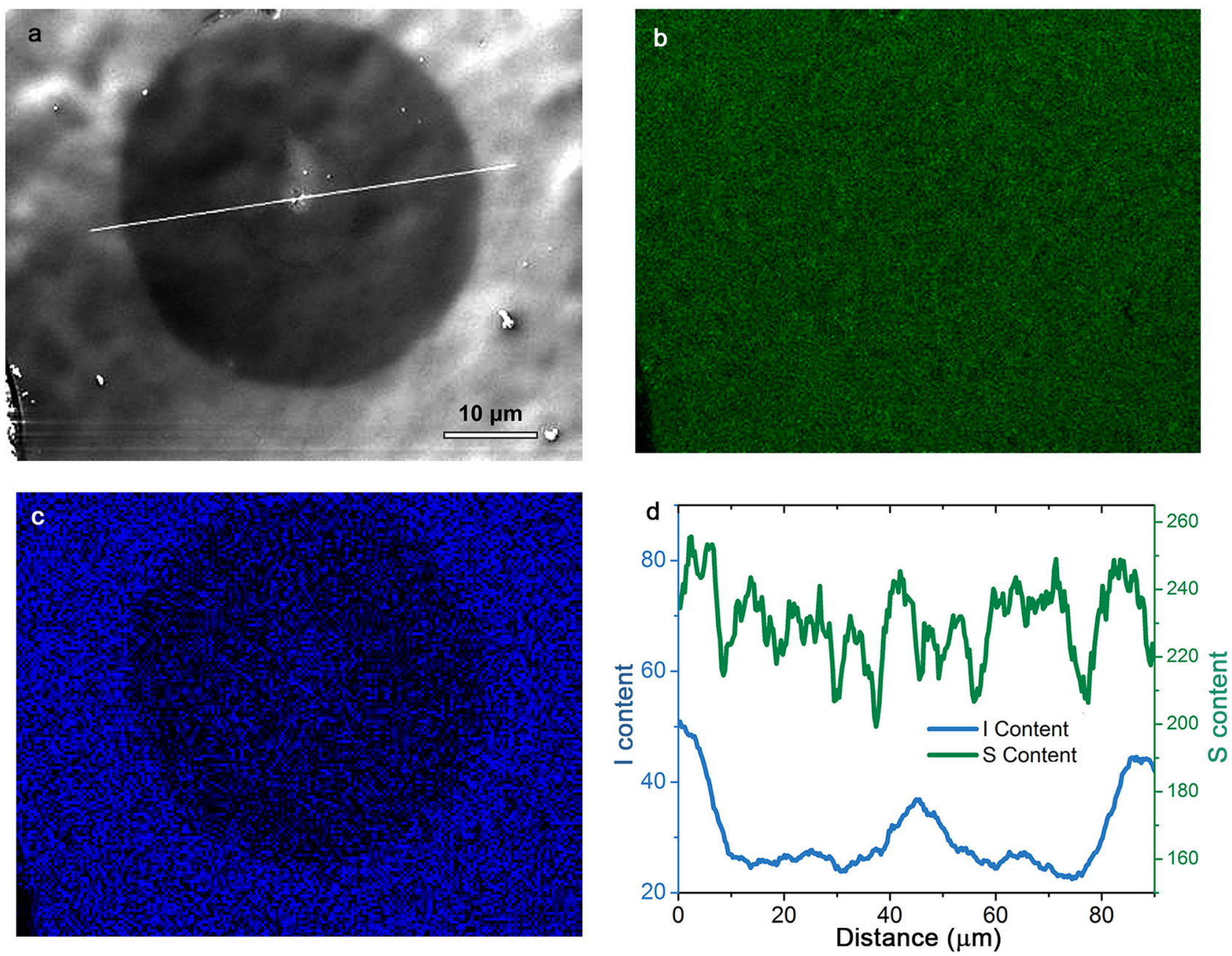

Fig. 4 SEM and EDX analysis of the (BEDO-TTF) $)_{2.4} \mathrm{l}_{3}$ after positive voltage bias application. a SEM image showing the discolored region around the Pt-tip and the EDX linear-scan range (white line). EDX elemental maps showing the distribution of $\mathbf{b}$ sulfur and $\mathbf{c}$ iodine contents of the film. d lodine and sulfur content counts along the linear-scan path. It is observed that the iodine content in the darker region seen in part (a) is much less than the background. lodine accumulation around the Pt-tip contact is also observed

near the electrode and is deficient in most part of the colorless zone at where the iodine concentration is around half of that in the colored zone (Fig. 4c, d); and (iii) outside of the colorless area the iodide content is uniform. In essence, the results demonstrate that when the electrical field is applied in moist environment, the triiodine anion in the metallic (BEDO-TTF) $)_{2.4} \mathrm{l}_{3}$ is depleted near the anode.

Further EDX analysis was carried out to obtain the approximate stoichiometry of the salts in various areas of the sample (position 1-3), including at the Pt electrode (position 4) after the positive bias application (Fig. 5). At position 1 (opaque area), the iodine to sulfur atomic percentage (At\%) ratio is determined to be $20.27 \%$ to $79.73 \%$ which agrees with the stoichiometry of (BEDO-TTF $)\left._{2.4}\right|_{3}$. At position 2 (colorless area), a clear decrease in iodine content is observed as also shown in Fig. 4d previously. Deeper inside the colorless zone (position 3), the iodine to sulfur At\% ratio is found to be $12.38 \%$ to $87.62 \%$, close to that expected for [(BEDOTTF $\left.)_{1.6}{ }^{+}(\mathrm{I})^{-}\left(\mathrm{H}_{2} \mathrm{O}\right)_{n}\right]$. This result is therefore consistent with the idea of $\mathrm{I}_{3}$ anions substitution by hydrated $\mathrm{I}$ anions in the colorless state. ${ }^{31}$ Consequently, the replacement of $\mathrm{I}_{3}{ }^{-}$anions by $\left[(\mathrm{I})^{-}\left(\mathrm{H}_{2} \mathrm{O}\right)_{n}\right]$ anions has a concomitant increase of the oxidation state of BEDO-TTF molecules from +0.42 in [(BEDO-TTF $\left.)_{2.4}{ }^{+}\left(\mathrm{I}_{3}\right)^{-}\right]$ to +0.63 in [(BEDO-TTF $\left.)_{1.6}{ }^{+}(\mathrm{I})^{-}\left(\mathrm{H}_{2} \mathrm{O}\right)_{n}\right]$. We suggest that the iodine, which is produced by the oxidation of iodide ions during a positive bias scan, is able to additionally oxidize BEDO-TTF molecules while its excess is reversibly adsorbed by the Ptbased electrode. ${ }^{34}$ Such ion-trapping mechanism was previously observed for other electrochromic materials showing a high degree of reversibility, as also in our case (ESI, see Figure ESI6). ${ }^{36}$ Indeed, at the Pt electrode (Fig. 5, position 4) a high concentration of iodine is observed. The iodine to sulfur atomic ratio is $66.76 \%$ to $33.24 \%$. In terms of the weight \% ratio, it is observed to be $88.83 \%$ to $11.17 \%$. The presence of sulfur at the Pt electrode may indicate a small amount of residual crystal that is being peeled-off and transferred from the film to the tip due to the hard Pt tip pressing onto the soft film.

Based on the observed results, we propose a two-step transformation of the anionic layer as the main mechanism for the removability of $\mathrm{I}_{3}{ }^{-}$anions that takes place under a positive potential scan. In the first step, $\mathrm{I}_{3}{ }^{-}$anions move to the positively charged Pt-based electrode, where they are oxidized to $\mathrm{I}_{2}$ molecules; some amount of iodine is now being trapped by the Pt-based anode. ${ }^{33}$ In the second step, the iodine that are not adsorbed, diffuse into the BEDO-TTF-based layer and react with BEDO-TTF molecules. In the presence of water this redox reaction results in the formation of the colorless anionic $\left[\mathrm{I}^{-}\left(\mathrm{H}_{2} \mathrm{O}\right)_{n}\right]$-based layer. Physically, at $\mathrm{RH}>85 \%$, the metallic [(BEDO-TTF $)_{2.4}{ }^{+}\left(\mathrm{I}_{3}\right)^{-}$]based layer is able to absorb enough water to organize the diffusive medium that mediates the movement of ions and molecules. During the anionic layer transformation the water 

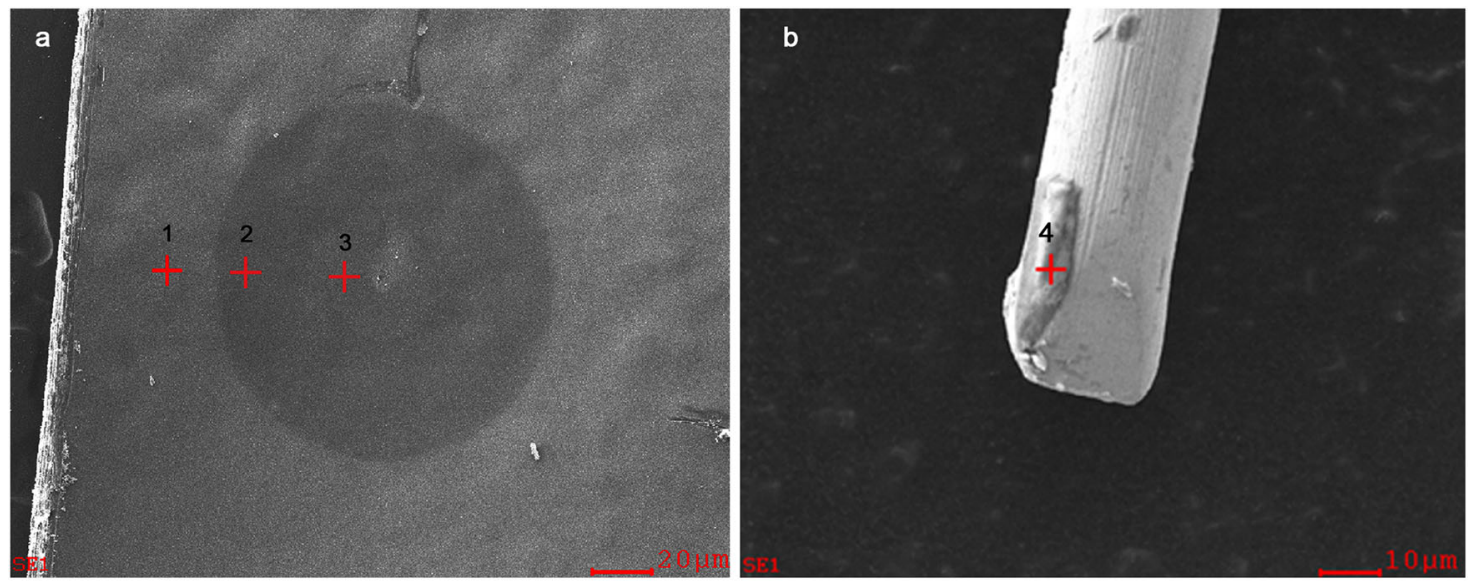

C

\begin{tabular}{lcccc}
\hline Position & 1 & 2 & 3 & 4 \\
Iodine (At\%) & 20.27 & 11.18 & 12.38 & 66.76 \\
Sulfur (At\%) & 79.73 & 88.82 & 87.62 & 33.24 \\
\hline
\end{tabular}

Fig. 5 EDX point-scan analysis of (BEDO-TTF $)_{2.4} l_{3}$ film and the platinum electrode after positive bias application. $\mathbf{a}(\text { BEDO-TTF })_{2.4} l_{3}$ film and $\mathbf{b}$ Pt electrode SEM images. The red plus sign indicates the EDX point-scan areas. c Tabulated experimental data of the atomic percentage of iodine and sulfur content at the four positions

molecules perform two important functions: (1) water facilitates both the transport of $\mathrm{I}_{3}{ }^{-}$anions to anode and the diffusion of the formed $\mathrm{I}_{2}$ molecules to the BEDO-TTF-based layer and (2) it helps to stabilize $\mathrm{I}^{-}$species via the formation of hydrated anions $\left[\left(I^{-}\left(\mathrm{H}_{2} \mathrm{O}\right)_{n}\right]\right.$ (see Eq. 1).

$$
\begin{gathered}
2\left[(\mathrm{BEDO}-\mathrm{TTF})_{2.4}^{+}\left(\mathrm{I}_{3}\right)^{-}\right](\text {dark }- \text { colored })-2 e^{-} \stackrel{n \mathrm{H}_{2} \mathrm{O}}{\longrightarrow} 4.8(\mathrm{BEDO}-\mathrm{TTF})+3 \mathrm{I}_{2} \\
\stackrel{n \mathrm{H}_{2} \mathrm{O}}{\longrightarrow} 3\left[(\mathrm{BEDO}-\mathrm{TTF})_{1.6}^{+}(\mathrm{I})^{-}\left(\mathrm{H}_{2} \mathrm{O}\right)_{\mathrm{n}}\right](\text { colorless })+1.5 \mathrm{I}_{2}(\text { adsorbed by Pt electrode })
\end{gathered}
$$

The removability and recovery of $\mathrm{I}_{3}{ }^{-}$anions is likely the unifying factor that is responsible for the electrochromic and diode-like behaviors of the studied BL film. The removability of $\mathrm{I}_{3}{ }^{-}$anions in moist environment effectively results in both the physical discoloration and also the change in the degree of charge transfer between BEDO-TTF molecules and iodide-based counter ion, turning the colorless area into a semiconducting barrier that is essential to achieve a diode-like property. ${ }^{4}$

It is remarkable that a thin bilayer film of (BEDO-TTF) ${ }_{2.4} I_{3} /$ polycarbonate can provide flexibility, reversibility, electrochromism, and rectifying behaviors all in a single relatively straightforward device due to a simple $\mathrm{I}_{3}{ }^{-}$migration processes. The reversible migration of iodine species and chemical transformation in a moist environment can be controlled by applying different bias to the $\mathrm{Pt}$ electrodes, effectively altering the coloration and the charge transfers. The reported results constitute a proof-of-concept that opens up new possibilities for the design and fabrication of organic electrochromic and rectifying devices that operate with a very simple working principle. Furthermore, the basic requirement for achieving such electrochromic behavior is attained without the need of using a complicated pre-processing or post-processing, since it simply needed a metallic electrode, a low voltage bias, and a small water content. We hope that our work will inspire others to prepare novel materials with lower operation voltages, high switching speeds, and better diode on/off current ratios. We are currently underway along these directions by investigating other conducting charge-transfer salts and electrodes combinations. Of interest would be the incorporation of electrode patterning techniques since they should allow a pixel by pixel manipulation of the film's transparency and a parallel device production.

\section{METHODS}

Bilayer films preparation

Samples of metallic BL polycarbonate/[(BEDO-TTF $n_{n}$ films were fabricated using the previously reported synthetic procedure..$^{5,10,26}$ Polymeric films were cast on a glass support at $130^{\circ} \mathrm{C}$ from a 1,2-dichlorobenzene solution of polycarbonate and the organic donor BEDO-TTF. Then, to cover the films with the (001)-oriented layers of (BEDO-TTF) $2.4 l_{3}$, we exposed the film surfaces to the vapors of a saturated solution of $\mathrm{I}_{2}$ in dichloromethane at equilibrium for $3 \mathrm{~min}$ at $30{ }^{\circ} \mathrm{C}$ and $\mathrm{RH}$ of $40 \%$. To control the temperature and humidity, a climatic chamber Memmert HPP 108 was used.

\section{Electronic characterization}

A piece of a bilayer film was cut and mounted on a G10 platform. Two 24 micron Pt wire were slightly pushed on their tips against the bilayer film to form two electrical contacts. The setup was then put inside a clear custombuilt humidity chamber positioned under an optical microscope with a digital camera recorder. The tests were performed using a Keithley 6517A electrometer and a HP 4156A semiconductor parameter analyzer.

\section{Temperature dependent transport}

Both the opaque and transparent films were first cut into size. Then, 12 micron gold wires were attached on the films using carbon paste electrodes at 4 equidistant positions. Measurements were carried out in 4point configuration using Keithley 6221 current source and Keithley 2182A nanovoltmeter.

\section{X-ray diffraction analysis}

Diffraction measurements were carried out on a Bruker D8Advance diffractometer with a CuKa radiation $(\lambda=1.54184 \AA \AA)$ with a Vantec-500 two dimensional detector and collimator of $0.5 \mathrm{~mm}$ on the primary optic. 2Theta scans were collected in a range from 4 to 42 degrees, $1200 \mathrm{~s} /$ frame. The BL film was mounted as it is on the sample holder. The conducting layer of the polycarbonate/(BEDO-TTF) $\left.{ }_{2.4}\right|_{3}$ was then directly subjected to obtain the $x$-ray pattern. We used the Bruker software package for 2D image processing and $2 \mathrm{D}$ diffraction pattern integration. 


\section{Raman characterization}

Micro-Raman measurements were carried out using the $5145 \AA$ line of an Argon-ion laser at room temperature with a Jobin-Yvon T-64000 Raman spectrometer attached to an Olympus microscope to allow for the focusing of the laser spot on the sample within a micrometer scale; and equipped with a liquid-nitrogen-cooled charge coupled device detector. Low laser power levels onto the sample were used to avoid degradation on the films due to overheating. The data acquisition was carried out with a spectral resolution of $1 \mathrm{~cm}^{-1}$. Silicon phonon-line $\left(521 \mathrm{~cm}^{-1}\right)$ was used for the calibration of the spectrometer.

\section{SEM and EDX study}

SEM and EDX measurements were obtained by field-emission JEOL JSM $7410 \mathrm{~F}$ at $5 \mathrm{keV}$ and $20 \mathrm{keV}$, respectively on uncoated films after positive bias application at high humidity levels.

\section{AFM testing}

The current atomic force microscopy images were acquired using a Keysight 5500 AFM with a current-to-voltage converter "Resiscope". The following tip RMN-25PT300 was used for all the scans. In order to acquire the current map, the sample was biased with a constant voltage throughout the measurement. To obtain the I/ $\mathrm{V}$ curves, the bias was swept with a slew rate of $15 \mathrm{~V} / \mathrm{s}$. A constant force of $200 \mathrm{nN}$ was applied to the sample in order to maintain a good mechanical contact.

\section{DATA AVAILABILITY}

The datasets supporting the current study are available from the corresponding author on reasonable request.

\section{ACKNOWLEDGEMENTS}

The authors acknowledge the financial support from NSF-DMR 1309146. We also acknowledge the financial support from Instituto de Salud Carlos III, through "Acciones CIBER." The Networking Research Center on Bioengineering, Biomaterials and Nanomedicine (CIBER-BBN), an initiative funded by the VI National R\&D\&I Plan 2008e2011, Iniciativa Ingenio 2010, Consolider Program, CIBER Actions and financed jDevelopment Fund. The authors also appreciate the financial support through projects: FANCY (CTQ2016-80030-R), MOTHER (MAT2016-80826-R), and MAT201451778-C2-1-R, granted by DGI (Spain), GenCat (2014-SGR-17, 2015 SGR 753, and 2017-SGR-918), financed by DGR (Catalunya), and Severo Ochoa Program Grant SEV2015-0496, financed by Mineco (Spain). A portion of this work was performed at the National High Magnetic Field Laboratory, which is supported by the National Science Foundation Cooperative Agreement No. DMR-1157490 and the State of Florida. This article is dedicated to the memory of Prof. Adam Tracz.

\section{AUTHOR CONTRIBUTIONS}

D.S. and E.L. designed and prepared the samples. E.L. has initiated and conducted the main line of this research. D.S. and E.S. performed the macroscopic experiments, E.D. X. data and recorded the video. E.L., E.S., J.V., and A.G. discussed results and wrote the manuscript. A.C. performed the DRX data measurements, N.M. performed the Raman study. A.G. performed the CSAFM measurements. C.R., E.S.C., and J.V. collaborated with the redaction and interpretation of the data.

\section{ADDITIONAL INFORMATION}

Supplementary information accompanies the paper on the npj Flexible Electronics website (https://doi.org/10.1038/s41528-018-0041-1).

Competing interests: The authors declare no competing interests.

Publisher's note: Springer Nature remains neutral with regard to jurisdictional claims in published maps and institutional affiliations.

\section{REFERENCES}

1. Akoudad, S. \& Roncali, J. Electrochemical synthesis of poly(3,4-ethylenedioxythiophene) from a dimer precursor. Synth. Met. 93, 111-114 (1998).
2. Kang, J. H. et al. A dual-polymer electrochromic device with high coloration efficiency and fast response time: Poly(3,4-(1,4-butylene-(2-ene)dioxy)thiophene)polyaniline ECD. Chem. - Asian J. 6, 2123-2129 (2011).

3. Bakhshi, A. K. \& Bhalla, G. Electrically conducting polymers: Materials of the twenty first century. J. Sci. Ind. Res. 63, 715-728 (2004).

4. Bassett, R. J. \& Taylor, P. D. Power Semiconductor Devices. in Electrical Engineer's Reference Book: 16th edn. 1-37 (2003). https://doi.org/10.1016/B978-0750646376/50017-4

5. Zhang, J. et al. Flexible indium-gallium-zinc-oxide Schottky diode operating beyond $2.45 \mathrm{GHz}$. Nat. Commun. 6, 7561 (2015).

6. Runnerstrom, E. L., Llordés, A., Lounis, S. D. \& Milliron, D. J. Nanostructured electrochromic smart windows: traditional materials and NIR-selective plasmonic nanocrystals. Chem. Commun. 50, 10555-10572 (2014).

7. Mortimer, R. J., Rosseinsky, D. R. \& Monk, P. M. S. Electrochromic materials and devices. Electro. Mater. Devices 77, 1-638 (2015).

8. Zou, P. et al. Bright and fast multicoloured voltage reporters via electrochromic FRET. Nat. Commun. 5(1), 4625 (2014).

9. Cong, S., Tian, Y., Li, Q., Zhao, Z. \& Geng, F. Single-crystalline tungsten oxide quantum dots for fast pseudocapacitor and electrochromic applications. Adv. Mater. 26, 4260-4267 (2014).

10. Patra, A., Bendikov, M. \& Chand, S. Poly(3,4-ethylenedioxyselenophene) and its derivatives: Novel organic electronic materials. Acc. Chem. Res. 47, 1465-1474 (2014).

11. Malavé Osuna, R., Hernández, V., López Navarrete, J. T., Kauppinen, E. I. \& Ruiz, V. Ultrafast and high-contrast electrochromism on bendable transparent carbon nanotube electrodes. J. Phys. Chem. Lett. 1, 1367-1371 (2010).

12. Sani, N. et al. All-printed diode operating at $1.6 \mathrm{GHz}$. Proc. Natl Acad. Sci. 111, 11943-11948 (2014).

13. Cambridge University Engineering Department. Materials data sources. Mater. Des. 9, 305 (1988).

14. Laukhin, V., Lebedev, V., Laukhina, E., Rovira, C. \& Veciana, J. Highly sensitive multi-layer pressure sensor with an active nanostructured layer of an organic molecular metal. IOP Conf. Ser. Mater. Sci. Eng. 108, 012038 (2016).

15. Laukhina, E. E. et al. Superconductivity in reticulate doped polycarbonate films, containing (BEDT-TTF)213. Synth. Met. 70, 797-800 (1995).

16. Lebedev, V., Laukhina, E., Laukhin, V., Rovira, C. \& Veciana, J. Tuning the electronic properties of piezoresistive bilayer films based on a-(BEDT-TTF) ${ }_{2} l_{3}$. Eur. J. Inorg. Chem. 2014, 3927-3932 (2014).

17. Pfattner, R. et al. A highly sensitive pyroresistive all-organic infrared bolometer. Adv. Electron. Mater. 1, 1-6 (2015).

18. Steven, E. et al. Silk/molecular conductor bilayer thin-films: Properties and sensing functions. Mater. Horiz. 1, 522-528 (2014).

19. Laukhina, E. et al. Ultrasensitive piezoresistive all-organic flexible thin films. Adv. Mater. 22, 977-981 (2010)

20. Shibaeva, R. P. \& Yagubskii, E. B. Molecular conductors and superconductors based on trihalides of BEDT-TTF and some of its analogues. Chem. Rev. 104, 5347-5378 (2004)

21. Jones, W. Organic molecular solids. Properties and applications. CRC Press, (1997).

22. Haneda, T., Tracz, A., Saito, G. \& Yamochi, H. Continuous and discontinuous water release/intake of (BEDO-TTF $)_{2} \mathrm{Br}\left(\mathrm{H}_{2} \mathrm{O}\right)_{3}$ micro-crystals embedded in polymer film. J. Mater. Chem. 21, 1621 (2011).

23. Balint, R., Cassidy, N. J. \& Cartmell, S. H. Conductive polymers: Towards a smart biomaterial for tissue engineering. Acta Biomater. 10, 2341-2353 (2014).

24. Zhang, X., Bäuerle, P., Aida, T., Skabara, P. \& Kagan, C. Organic electronics for a better tomorrow: Innovation, accessibility, sustainability. A White Pap. from Chem. Sci. Soc. Summit 34 (2012).

25. Lebedev, V. et al. Investigation of sensing capabilities of organic bi-layer thermistor in wearable e-textile and wireless sensing devices. Org. Electron. Phys., Mater. Appl. 42, 146-152 (2017).

26. Sekitani, T., Zschieschang, U., Klauk, H. \& Someya, T. Flexible organic transistors and circuits with extreme bending stability. Nat. Mater. 9, 1015-1022 (2010).

27. Wudl, F. et al. BEDO)2,4l3: the first robust organic metal of BEDO-TTF. J. Am. Chem. Soc. 112, 2461-2462 (1990).

28. Hornchi, S. et al. Highly-oriented BEDO-TTF molecules in metallic polymer composites. Mol. Cryst. Liq. Cryst. 296, 365-382 (1997).

29. Jeszka, J. K. Metallic polymer composites with bis(ethylenedioxy)-tetrathiafulvalene salts. Preparation-properties relationship. Synth. Met 106, 75-83 (1999).

30. Tracz, A. et al. New transparent, colorless, metallically conductive polymer films and their electrochemical transformations. Synth. Met. 86, 2173-2174 (1997).

31. Tracz, A. lon exchange in $(\mathrm{BO})_{2} \cdot 4 I_{3}$ microcrystals: A method for obtaining colorless, transparent, metallically conductive polymer films. J. Appl. Polym. Sci. 86, 1465-1472 (2002) 
32. Zorina, L. V., Simonov, S. V., Khasanov, S. S. \& Shibaeva, R. P. New low-dimensional molecular conductors: $a^{\prime \prime}-(\mathrm{BEDO}-\mathrm{TTF})_{2} \mathrm{Cl} \cdot 3 \mathrm{H}_{2} \mathrm{O}$ and $\theta-(\mathrm{BDH}-\mathrm{TTP})_{2} \quad\left(\mathrm{Br}_{0.67}\right.$ $\left.\mathrm{Cl}_{0.33}\right) \cdot 3 \mathrm{H}_{2} \mathrm{O}$. Low. Temp. Phys. 37, 744-748 (2011).

33. Liao, X., Wang, J., Li, G. \& He, J. Effect of supercritical carbon dioxide on the crystallization and melting behavior of linear bisphenol a polycarbonate. J. Polym. Sci. Part B Polym. Phys. 42, 280-285 (2004).

34. Shu, Z. X. \& Bruckenstein, S. lodine adsorption studies at platinum. J. Electroanal. Chem. 317, 263-277 (1991).

35. Song, A. M. Nanometer-scale two-terminal semiconductor memory operating at room temperature. Appl. Phys. Lett. 86(4), 042106 (2005).

36. Wen, R. T., Granqvist, C. G. \& Niklasson, G. A. Eliminating degradation and uncovering ion-trapping dynamics in electrochromic WO 3 thin films. Nat. Mater. 14, 996-1001 (2015).

37. Moser, M. L. et al. Fast electrochromic device based on single-walled carbon nanotube thin films. Nano Lett. 16, 5386-5393 (2016).

38. $\mathrm{Xu}, \mathrm{T}$. et al. High-contrast and fast electrochromic switching enabled by plasmonics. Nat. Commun. 7, 10479 (2016).

39. Sapp, S. A., Sotzing, G. A., Reddinger, J. L. \& Reynolds, J. R. Rapid switching solid state electrochromic devices based on complementary conducting polymer films. Adv. Mater. 8, 808-811 (1996).
Open Access This article is licensed under a Creative Commons Attribution 4.0 International License, which permits use, sharing, adaptation, distribution and reproduction in any medium or format, as long as you give appropriate credit to the original author(s) and the source, provide a link to the Creative Commons license, and indicate if changes were made. The images or other third party material in this article are included in the article's Creative Commons license, unless indicated otherwise in a credit line to the material. If material is not included in the article's Creative Commons license and your intended use is not permitted by statutory regulation or exceeds the permitted use, you will need to obtain permission directly from the copyright holder. To view a copy of this license, visit http://creativecommons. org/licenses/by/4.0/.

(c) The Author(s) 2018 